ScIDoc Publishers

International Journal of Bioorganic Chemistry \& Molecular Biology (IJBCMB)

\title{
Small-Angle Neutron Scattering (SANS) and Wide-Angle X-Ray Diffraction (WAXD) Comparative Study on Malignant and Benign Human Cancer Cells and Tissues under Synchrotron Radiation
}

\section{Alireza Heidari*}

Faculty of Chemistry, California South University, 14731 Comet St. Irvine, CA 92604, USA.

In the current study, we have experimentally and comparatively investigated and compared malignant human cancer cells and tissues before and after irradiating of synchrotron radiation using Small-Angle Neutron Scattering (SANS) and Wide-Angle X-Ray
Diffraction (WAXD), It is clear that malignant human cancer cells and tissues have gradually transformed to benign human cancer cells and tissues under synchrotron radiation with the passing of time (Figures 1 and 2) [1-135].

Figure 1. Small-Angle Neutron Scattering (SANS) analysis of malignant human cancer cells and tissues (a) before and (b) after irradiating of synchrotron radiation in transformation process to benign human cancer cells and tissues with the passing of time [1-135].

(a)

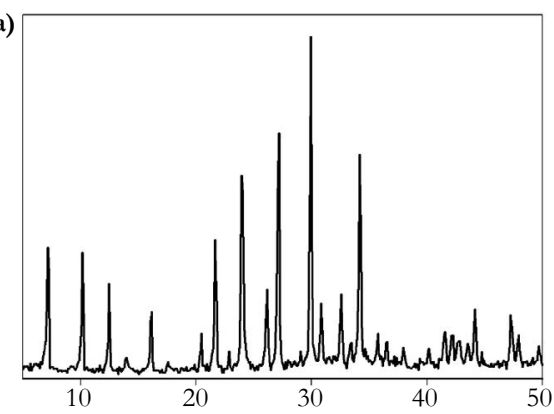

(b)

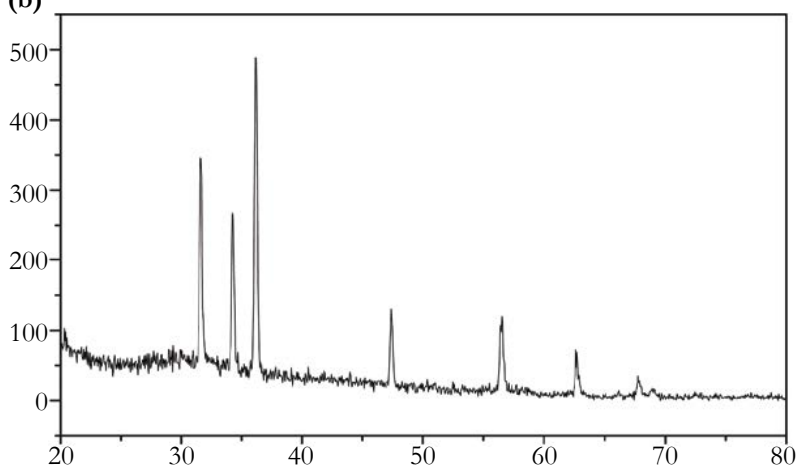

*Corresponding Author:

Alireza Heidari,

Faculty of Chemistry, California South University, 14731 Comet St. Irvine, CA 92604, USA.

E-mail: Scholar.Researcher.Scientist@gmail.com

Received: February 24, 2018

Published: March 01, 2018

Citation: Heidari A. Small-Angle Neutron Scattering (SANS) and Wide-Angle X-Ray Diffraction (WAXD) Comparative Study on Malignant and Benign Human Cancer Cells and Tissues under Synchrotron Radiation. Int J Bioorg Chem Mol Biol. 2018;6(2e):1-6. doi: http://dx.doi.org/10.19070/2332-2756-180009e

Copyright: Heidari $\mathbf{A}^{\circ}$ 2018. This is an open-access article distributed under the terms of the Creative Commons Attribution License, which permits unrestricted use, distribution and reproduction in any medium, provided the original author and source are credited. 
Figure 2. Wide-Angle X-Ray Diffraction (WAXD) analysis of malignant human cancer cells and tissues (a) before and (b) after irradiating of synchrotron radiation in transformation process to benign human cancer cells and tissues with the passing of time [1-135].

(a)
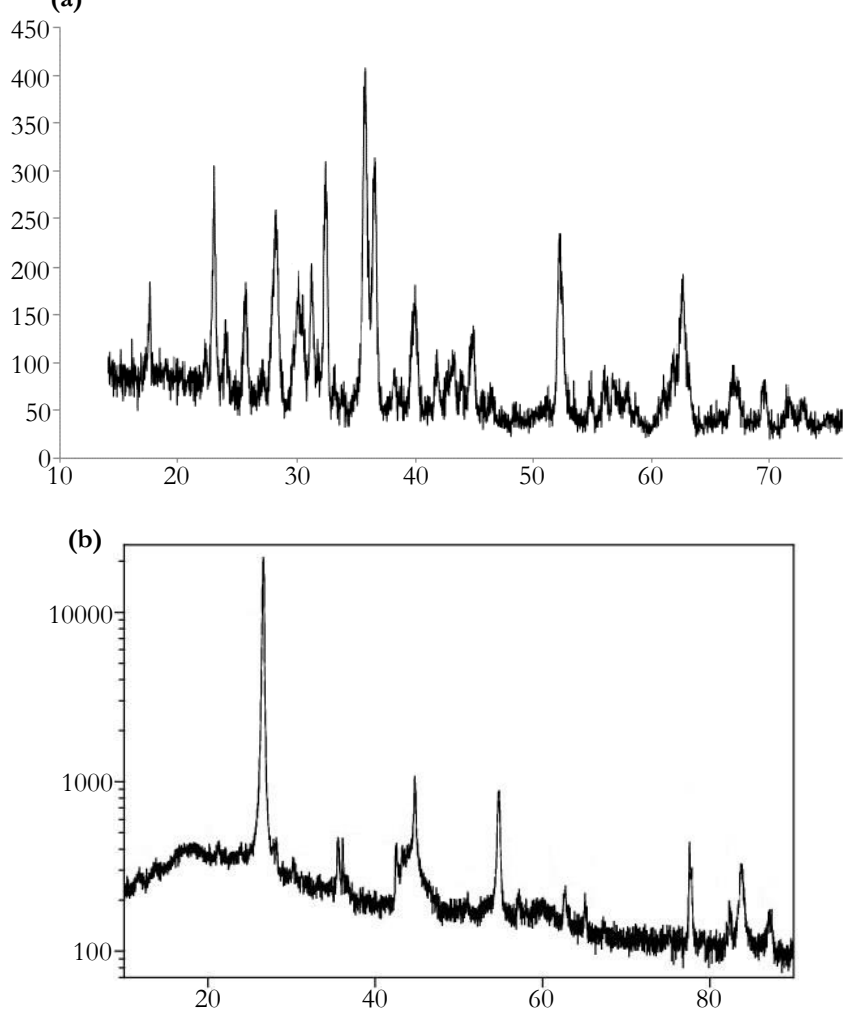

It can be concluded that malignant human cancer cells and tissues have gradually transformed to benign human cancer cells and tissues under synchrotron radiation with the passing of time (Figures 1 and 2) [1-135].

\section{References}

[1]. Heidari A, Brown C. Study of composition and morphology of cadmium oxide $(\mathrm{CdO})$ nanoparticles for eliminating cancer cells. J Nanomed Res. 2015;2(5):1-20.

[2]. Heidari A, Brown C. Study of Surface Morphological, Phytochemical and Structural Characteristics of rhodium (III) oxide (Rh2O3) nanoparticles. International Journal of Pharmacology, Phytochemistry and Ethnomedicine. 2015;1:15-9.

[3]. Heidari A. An Experimental Biospectroscopic Study on Seminal Plasma in Determination of Semen Quality for Evaluation of Male Infertility. Int J Adv Technol. 2016;7(2):1-2.

[4]. Heidari A. Extraction and Preconcentration of N-Tolyl-Sulfonyl-Phosphoramid-Saeure-Dichlorid as an Anti-Cancer Drug from Plants: A Pharmacognosy Study. J Pharmacogn Nat Prod. 2016;2:e103.

[5]. Heidari A. A Thermodynamic study on hydration and dehydration of DNA and RNA- amphiphile Complexes. J Bioeng Biomed Sci S. 2016;6.

[6]. Heidari A. Computational Studies on Molecular Structures and Carbonyl and Ketene Groups' Effects of Singlet and Triplet Energies of Azidoketene $\mathrm{O}=\mathrm{C}=\mathrm{CH}-\mathrm{NNN}$ and Isocyanatoketene $\mathrm{O}=\mathrm{C}=\mathrm{CH}-\mathrm{N}=\mathrm{C}=\mathrm{O}$. J Appl Computat Math. 2016;5(1):e142.

[7]. Heidari A. Study of Irradiations to Enhance the Induces the Dissociation of Hydrogen Bonds between Peptide Chains and Transition from Helix Structure to Random Coil Structure Using ATR-FTIR, Raman and 1HNMR Spectroscopies. J Biomol Res Ther. 2016;5(2):e146.

[8]. Heidari A. Future Prospects of Point Fluorescence Spectroscopy, Fluorescence Imaging and Fluorescence Endoscopy in Photodynamic Therapy (PDT) for Cancer Cells. J Bioanal Biomed. 2016;8(2):e135.

[9]. Heidari A. A Bio-Spectroscopic Study of DNA Density and Color Role as Determining Factor for Absorbed Irradiation in Cancer Cells. Adv Cancer Prev. 2016;1(2):e102.

[10]. Heidari A. Manufacturing Process of Solar Cells Using Cadmium Oxide
(CdO) and Rhodium (III) Oxide (Rh2O3) Nanoparticles. J Biotechno Biomater. 2016;6(2):e125.

[11]. Heidari A. A Novel Experimental and Computational Approach to Photobiosimulation of Telomeric DNA/RNA: A Biospectroscopic and Photobiological Study. J Res Development. 2016;4(1):1000144.

[12]. Alireza Heidari. Biochemical and Pharmacodynamical Study of Microporous Molecularly Imprinted Polymer Selective for Vancomycin, Teicoplanin, Oritavancin, Telavancin and Dalbavancin Binding. Biochem Physiol. 2016;5:e146.

[13]. Heidari A. Anti-Cancer Effect of UV Irradiation at Presence of Cadmium Oxide (CdO) Nanoparticles on DNA of Cancer Cells: A Photodynamic Therapy Study. Arch Cancer Res. 2016;4(1).

[14]. Heidari A. Biospectroscopic Study on Multi-Component Reactions (MCRs) in Two A-Type and B-Type Conformations of Nucleic Acids to Determine Ligand Binding Modes, Binding Constant and Stability of Nucleic Acids in Cadmium Oxide (CdO) Nanoparticles-Nucleic Acids Complexes as AntiCancer Drugs. Arch Cancer Res. 2016;4(2):65.

[15]. Heidari A. Simulation of Temperature Distribution of DNA/RNA of $\mathrm{Hu}$ man Cancer Cells Using Time-Dependent Bio-Heat Equation and $\mathrm{Nd}$ : YAG Lasers. Arch Cancer Res. 2016;4(2):69.

[16]. Heidari A. Quantitative Structure-Activity Relationship (QSAR) Approximation for Cadmium Oxide (CdO) and Rhodium (III) Oxide (Rh2 O3) Nanoparticles as Anti-Cancer Drugs for the Catalytic Formation of Proviral DNA from Viral RNA Using Multiple Linear and Non-Linear Correlation Approach. Ann Clin Lab Res. 2016;4(1).

[17]. Heidari A. Biomedical Study of Cancer Cells DNA Therapy Using Laser Irradiations at Presence of Intelligent Nanoparticles. J Biomedical Sci. 2016;5(2):9.

[18]. Heidari A. Measurement the Amount of Vitamin D2 (Ergocalciferol), Vitamin D3 (Cholecalciferol) and Absorbable Calcium (Ca2+), Iron (II)(Fe2+), Magnesium ( $\mathrm{Mg} 2+)$, Phosphate (PO4-) and Zinc ( $\mathrm{Zn2+}$ ) in Apricot Using High-Performance Liquid Chromatography (HPLC) and Spectroscopic Techniques. J Biom Biostat. 2016;7(2):292.

[19]. Heidari A. Spectroscopy and Quantum Mechanics of the Helium Dimer $(\mathrm{He} 2+)$, Neon Dimer (Ne2+), Argon Dimer (Ar2+), Krypton Dimer $(\mathrm{Kr} 2+)$, Xenon Dimer $(\mathrm{Xe} 2+)$, Radon Dimer $(\mathrm{Rn} 2+)$ and Ununoctium Dimer (Uuo2+) Molecular Cations. Chem Sci J. 2016;7:e112.

[20]. Heidari A. Human Toxicity Photodynamic Therapy Studies on DNA/ RNA Complexes as a Promising New Sensitizer for the Treatment of Malignant Tumors Using Bio-Spectroscopic Techniques. J Drug Metab Toxicol. 
2016;7(2):e129.

[21]. Heidari A. Novel and Stable Modifications of Intelligent Cadmium Oxide (CdO) Nanoparticles as Anti-Cancer Drug in Formation of Nucleic Acids Complexes for Human Cancer Cells' Treatment. Biochem Pharmacol (Los Angel). 2016;5(3):207

[22]. Heidari A. A Combined Computational and QM/MM Molecular Dynamics Study on Boron Nitride Nanotubes (BNNTs), Amorphous Boron Nitride Nanotubes (a-BNNTs) and Hexagonal Boron Nitride Nanotubes (h-BNNTs) as Hydrogen Storage. Struct Chem Crystallogr Commun. 2016;2(1):7.

[23]. Heidari A. Pharmaceutical and Analytical Chemistry Study of Cadmium Oxide (CdO) Nanoparticles Synthesis Methods and Properties as AntiCancer Drug and its Effect on Human Cancer Cells. Pharm Anal Chem Open Access. 2016;2(2):113.

[24]. Heidari A. A Chemotherapeutic and Biospectroscopic Investigation of the Interaction of Double-Standard DNA/RNA-Binding Molecules with Cadmium Oxide (CdO) and Rhodium (III) Oxide (Rh2O3) Nanoparticles as Anti-Cancer Drugs for Cancer Cells' Treatment. Chemo Open Access. 2016;5(2):e129.

[25]. Heidari A. Pharmacokinetics and Experimental Therapeutic Study of DNA and Other Biomolecules Using Lasers: Advantages and Applications. J Pharmacokinet Exp Ther. 2016;1(1):e005.

[26]. Heidari A. Determination of Ratio and Stability Constant of DNA/RNA in Human Cancer Cells and Cadmium Oxide (CdO) Nanoparticles Complexes Using Analytical Electrochemical and Spectroscopic Techniques. Insights Anal Electrochem. 2016;2(1):14

[27]. Heidari A. Discriminate between Antibacterial and Non-Antibacterial Drugs Artificial Neutral Networks of a Multilayer Perceptron (MLP) Type Using a Set of Topological Descriptors. J Heavy Met Toxicity Dis. 2016;1(2).

[28]. Heidari A. Combined Theoretical and Computational Study of the Belousovâ Zhabotinsky Chaotic Reaction and Curtius Rearrangement for Synthesis of Mechlorethamine, Cisplatin, Streptozotocin, Cyclophosphamide, Melphalan, Busulphan and BCNU as Antiâ Cancer Drugs. Insights Med Phys. 2016 Jun 12;1(2)

[29]. Heidari A. A Translational Biomedical Approach to Structural Arrangement of Amino Acids Complexes: A Combined Theoretical and Computational Study. Translational Biomedicine. 2016 Jun 7;7(2).

[30]. Heidari A. Ab Initio and Density Functional Theory (DFT) Studies of Dynamic NMR Shielding Tensors and Vibrational Frequencies of DNA/RNA and Cadmium Oxide (CdO) Nanoparticles Complexes in Human Cancer Cells. J Nanomedine Biotherapeutic Discov. 2016;6(2):e144

[31]. Heidari A. Molecular Dynamics and Monte-Carlo Simulations for Replacement Sugars in Insulin Resistance, Obesity, LDL Cholesterol, Triglycerides, Metabolic Syndrome, Type 2 Diabetes and Cardiovascular Disease: A Glycobiological Study. J Glycobiol. 2016;5(1):e111.

[32]. Heidari A. Synthesis and Study of 5-[(Phenylsulfonyl) Amino]-1, 3, 4-Thiadiazole-2-Sulfonamide as Potential Anti-Pertussis Drug Using Chromatography and Spectroscopy Techniques. Transl Med (Sunnyvale). 2016;6(3):e137.

[33]. Heidari A. Nitrogen, Oxygen, Phosphorus and Sulphur Heterocyclic AntiCancer Nano Drugs Separation in the Supercritical Fluid of Ozone (O3) Using Soave-Redlich-Kwong (SRK) and Pang-Robinson (PR) Equations. Electronic J Biol. 2016;12(4).

[34]. Heidari A. An Analytical and Computational Infrared Spectroscopic Review of Vibrational Modes in Nucleic Acids. Austin J Anal Pharm Chem. 2016;3(1): 1058

[35]. Heidari A, Brown C. Phase, Composition and Morphology Study and Analysis of Os-Pd/HfC Nanocomposites. Nano Research \& Applications. 2016;2(1).

[36]. Heidari A, Brown C. Vibrational spectroscopic study of intensities and shifts of symmetric vibration modes of ozone diluted by cumene. International Journal of Advanced Chemistry. 2016 May 7;4(1):5-9.

[37]. Heidari A. Study of the Role of Anti-Cancer Molecules with Different Sizes for Decreasing Corresponding Bulk Tumor Multiple Organs or Tissues. Arch Can Res. 2016 Jul 23;4(2).

[38]. Heidari A. Genomics and Proteomics Studies of Zolpidem, Necopidem, Alpidem, Saripidem, Miroprofen, Zolimidine, Olprinone and Abafungin as Anti-Tumor, Peptide Antibiotics, Antiviral and Central Nervous System (CNS) Drugs. J Data Mining Genomics \& Proteomics. 2016;7(3):e125.

[39]. Heidari A. Pharmacogenomics and Pharmacoproteomics Studies of Phosphodiesterase-5 (PDE5) Inhibitors and Paclitaxel Albumin-Stabilized Nanoparticles as Sandwiched Anti-Cancer Nano Drugs between Two DNA/ RNA Molecules of Human Cancer Cells. J Pharmacogenomics Pharmacoproteomics. 2016;7(2):e153.

[40]. Heidari A. Biotranslational Medical and Biospectroscopic Studies of Cadmium Oxide (CdO) Nanoparticles-DNA/RNA Straight and Cycle Chain
Complexes as Potent Anti-Viral, Anti-Tumor and Anti-Microbial Drugs: A Clinical Approach. Transl Biomed. 2016;7(2):76.

[41]. Heidari A. A Comparative Study on Simultaneous Determination and Separation of Adsorbed Cadmium Oxide (CdO) Nanoparticles on DNA/RNA of Human Cancer Cells Using Biospectroscopic Techniques and Dielectrophoresis (DEP) Method. Arch Can Res. 2016;4(2):86.

[42]. Heidari A. Cheminformatics and System Chemistry of Cisplatin, Carboplatin, Nedaplatin, Oxaliplatin, Heptaplatin and Lobaplatin as Anti-Cancer Nano Drugs: A Combined Computational and Experimental Study. J Inform Data Min. 2016 Jul 7;1(3).

[43]. Heidari A. Linear and Non-Linear Quantitative Structure-Anti-CancerActivity Relationship (QSACAR) Study of Hydrous Ruthenium (IV) Oxide (RuO2) Nanoparticles as Non-Nucleoside Reverse Transcriptase Inhibitors (NNRTIs) and Anti-Cancer Nano Drugs. J Integr Oncol. 2016;5(3):e110.

[44]. Heidari A. Synthesis, Characterization and Biospectroscopic Studies of Cadmium Oxide (CdO) Nanoparticles-Nucleic Acids Complexes Absence of Soluble Polymer as a Protective Agent Using Nucleic Acids Condensation and Solution Reduction Method. J Nanosci Curr Res. 2016;1(1):e101.

[45]. Heidari A. Coplanarity and Collinearity of 4'-Dinonyl-2, 2'-Bithiazole in One Domain of Bleomycin and Pingyangmycin to be Responsible for Binding of Cadmium Oxide (CdO) Nanoparticles to DNA/RNA Bidentate Ligands as Anti-Tumor Nano Drug. Int J Drug Dev \& Res. 2016;8(3):01-2.

[46]. Heidari A. A Pharmacovigilance Study on Linear and Non-Linear Quantitative Structure (Chromatographic) Retention Relationships (QSRR) Models for the Prediction of Retention Time of Anti-Cancer Nano Drugs under Synchrotron Radiations. J Pharmacovigil. 2016;4(4):e161.

[47]. Heidari A. Nanotechnology in Preparation of Semipermeable Polymers. J Adv Chem Eng. 2016;6(2):157.

[48]. Heidari A. A Gastrointestinal Study on Linear and Non-Linear Quantitative Structure (Chromatographic) Retention Relationships (QSRR) Models for Analysis 5-Aminosalicylates Nano Particles as Digestive System Nano Drugs under Synchrotron Radiations. J Gastrointest Dig Syst. 2016;6(4):e119.

[49]. Heidari A. DNA/RNA Fragmentation and Cytolysis in Human Cancer Cells Treated with Diphthamide Nano Particles Derivatives. Biomedical Data Mining. 2016;5(1):e102.

[50]. Heidari A. A Successful Strategy for the Prediction of Solubility in the Construction of Quantitative Structure-Activity Relationship (QSAR) and Quantitative Structure-Property Relationship (QSPR) under Synchrotron Radiations Using Genetic Function Approximation (GFA) Algorithm. J Mol Biol Biotechnol. 2016;1(1):1

[51]. Heidari A. Computational Study on Molecular Structures of C20, C60, C240, C540, C960, C2160 and C3840 Fullerene Nano Molecules under Synchrotron Radiations Using Fuzzy Logic. J Material Sci Eng. 2016;5(282):2169-0022.

[52]. Heidari A. Graph Theoretical Analysis of Zigzag Polyhexamethylene Biguanide, Polyhexamethylene Adipamide, Polyhexamethylene Biguanide Gauze and Polyhexamethylene Biguanide Hydrochloride (PHMB) Boron Nitride Nanotubes (BNNTs), Amorphous Boron Nitride Nanotubes (aBNNTs) and Hexagonal Boron Nitride Nanotubes (h-BNNTs). J Appl Computat Math. 2016;5(5):e143.

[53]. Heidari A. The Impact of High Resolution Imaging on Diagnosis. Int J Clin Med Imaging. 2016;3:1000e101.

[54]. Heidari A. A Comparative Study of Conformational Behavior of Isotretinoin (13-Cis Retinoic Acid) and Tretinoin (All-Trans Retinoic Acid (ATRA)) Nano Particles as Anti-Cancer Nano Drugs under Synchrotron Radiations Using Hartree-Fock (HF) and Density Functional Theory (DFT) Methods. Insights in Biomed. 2016;2.

[55]. Heidari A. Advances in Logic, Operations and Computational Mathematics. J Appl Computat Math. 2016;5(5):e144.

[56]. Heidari A. Mathematical Equations in Predicting Physical Behavior. Journal of Applied \& Computational Mathematics. 2016;5(5).

[57]. Heidari A. Chemotherapy a Last Resort for Cancer Treatment. Chemo Open Access. 2016;5(4):e130.

[58]. Heidari A. Separation and Pre-Concentration of Metal Cations-DNA/ RNA Chelates Using Molecular Beam Mass Spectrometry with Tunable Vacuum Ultraviolet (VUV) Synchrotron Radiation and Various Analytical Methods. Mass Spectrom Purif Tech. 2016;2(1):e101.

[59]. Heidari A. Yoctosecond Quantitative Structure-Activity Relationship (QSAR) and Quantitative Structure-Property Relationship (QSPR) under Synchrotron Radiations Studies for Prediction of Solubility of Anti-Cancer Nano Drugs in Aqueous Solutions Using Genetic Function Approximation (GFA) Algorithm. Insight Pharm Res. 2016;1(1):6.

[60]. Heidari A. Cancer Risk Prediction and Assessment in Human Cells under Synchrotron Radiations Using Quantitative Structure Activity Relationship (QSAR) and Quantitative Structure Properties Relationship (QSPR) Studies. Int J Clin Med Imaging 3: 516. doi. 2016;10:2376-0249.

[61]. Heidari A. A Novel Approach to Biology. Electronic J Biol. 2016;12(4). 
[62]. Heidari A. Innovative Biomedical Equipment's for Diagnosis and Treatment. J Bioengineer \& Biomedical Sci. 2016;6(2).

[63]. Heidari A. Integrating Precision Cancer Medicine into Healthcare, Medicare Reimbursement Changes and the Practice of Oncology: Trends in Oncology Medicine and Practices. J Oncol Med \& Pract. 2016;1(2):e101.

[64]. Heidari A. Promoting Convergence in Biomedical and Biomaterials Sciences and Silk Proteins for Biomedical and Biomaterials Applications: An Introduction to Materials in Medicine and Bioengineering Perspectives. J Bioengineer \& Biomedical Sci. 2016;6(3)

[65]. Heidari A. X-Ray Fluorescence and X-Ray Diffraction Analysis on Discrete Element Modeling of Nano Powder Metallurgy Processes in Optimal Container Design. J Powder Metall Min. 2017;6(1).

[66]. Heidari A. Biomolecular Spectroscopy and Dynamics of Nano-Sized Molecules and Clusters as Cross-Linking-Induced Anti-Cancer and ImmuneOncology Nano Drugs Delivery in DNA/RNA of Human Cancer Cells' Membranes under Synchrotron Radiations: A Payload-Based Perspective. Arch Chem Res. 2017;1(2):11

[67]. Heidari A. Deficiencies in Repair of Double-Standard DNA/RNA-Binding Molecules Identified in Many Types of Solid and Liquid Tumors Oncology in Human Body for Advancing Cancer Immunotherapy Using Computer Simulations and Data Analysis. J Appl Bioinforma Comput Biol. 2017;6(1).

[68]. Heidari A. Electronic Coupling among the Five Nanomolecules Shuts Down Quantum Tunneling in the Presence and Absence of an Applied Magnetic Field for Indication of the Dimer or other Provide Different Influences on the Magnetic Behavior of Single Molecular Magnets (SMMs) as Qubits for Quantum Computing. Glob J Res Rev. 2017;4(2):69.

[69]. Heidari A. Polymorphism in Nano-Sized Graphene Ligand-Induced Transformation of Au38-xAgx/xCux (SPh-tBu) 24 to Au36-xAgx/xCux (SPhtBu) $24(\mathrm{x}=1-12)$ Nanomolecules for Synthesis of Au144-xAgx $/ x$ Cux [(SR) 60,(SC4) 60,(SC6) 60,(SC12) 60,(PET) 60,(p-MBA) 60,(F) 60,(Cl) 60,(Br) 60,(I) 60,(At) 60,(Uus) 60 and (SC6H13) 60] Nano. Journal of Nanomaterials \& Molecular Nanotechnology. 2017 Jun 9;2017.

[70]. Heidari A. Biomedical Resource Oncology and Data Mining to Enable Resource Discovery in Medical, Medicinal, Clinical, Pharmaceutical, Chemical and Translational Research and Their Applications in Cancer Research. Int J Biomed Data Min. 2017;6:e103.

[71]. Heidari A. Study of Synthesis, Pharmacokinetics, Pharmacodynamics, Dosing, Stability, Safety and Efficacy of Olympiadane Nanomolecules as Agent for Cancer Enzymotherapy, Immunotherapy. Chemotherapy, Radiotherapy, Hormone Therapy and Targeted. J Dev Drugs. 2017;6:e154.

[72]. Heidari A. A Novel Approach to Future Horizon of Top Seven Biomedical Research Topics to Watch in 2017: Alzheimer's, Ebola, Hypersomnia, Human Immunodeficiency Virus (HIV), Tuberculosis (TB), Microbiome/ Antibiotic Resistance and Endovascular Stroke. J Bioengineer \& Biomedical Sci. 2017;7:e127.

[73]. Heidari A. Opinion on Computational Fluid Dynamics (CFD) Technique. Fluid Mech Open Acc. 2017;4(157):2476-296.

[74]. Heidari A. Concurrent Diagnosis of Oncology Influence Outcomes in Emergency General Surgery for Colorectal Cancer and Multiple Sclerosis (MS) Treatment Using Magnetic Resonance Imaging (MRI) and Au329(SR)84, Au329-xAgx(SR)84, Au144(SR)60, Au68(SR)36, Au30(SR)18, Au102(SPh)44, Au38(SPh)24, Au38(SC2H4Ph)24, Au21S(SAdm)15, Au36(pMBA) 24 and Au25(pMBA) 18 Nano Clusters. J Surgery Emerg Med. 2017;21(1)

[75]. Heidari A. Developmental Cell Biology in Adult Stem Cells Death and Autophagy to Trigger a Preventive Allergic Reaction to Common Airborne Allergens under Synchrotron Radiation Using Nanotechnology for Therapeutic Goals in Particular Allergy Shots (Immunotherapy). Cell Biology: Research \& Therapy. 2017 Jun 27;2017.

[76]. Heidari A. Changing Metal Powder Characteristics for Elimination of the Heavy Metals Toxicity and Diseases in Disruption of Extracellular Matrix (ECM) Proteins Adjustment in Cancer Metastases Induced by Osteosarcoma, Chondrosarcoma, Carcinoid, Carcinoma, Ewing's Sarcoma, Fibrosarcoma and Secondary Hematopoietic Solid or Soft Tissue Tumors. J Powder Metall Min. 2017;6:170

[77]. Heidari A. Nanomedicine-Based Combination Anti-Cancer Therapy between Nucleic Acids and Anti-Cancer Nano Drugs in Covalent Nano Drugs Delivery Systems for Selective Imaging and Treatment of Human Brain Tumors Using Hyaluronic Acid, Alguronic Acid and Sodium Hyaluronate as Anti-Cancer Nano Drugs and Nucleic Acids Delivery under Synchrotron Radiation. American Journal of Advanced Drug Delivery. 2017 Jul 12;5(2).

[78]. Heidari A. Clinical Trials of Dendritic Cell Therapies for Cancer Exposing Vulnerabilities in Human Cancer Cells' Metabolism and Metabolomics: New Discoveries, Unique Features Inform New Therapeutic Opportunities, Biotech's Bumpy Road to the Market and Elucidating the Biochemical Programs that Support Cancer Initiation and Progression. J Biol Med Science. 2017;1:e103.
[79]. Heidari A. The Design Graphene-Based Nanosheets as a New Nanomaterial in Anti-Cancer Therapy and Delivery of Chemotherapeutics and Biological Nano Drugs for Liposomal Anti-Cancer Nano Drugs and Gene Delivery. Br Biomed Bull. 2017;5:305.

[80]. Heidari A. Integrative Approach to Biological Networks for Emerging Roles of Proteomics, Genomics and Transcriptomics in the Discovery and Validation of Human Colorectal Cancer Biomarkers from DNA/RNA Sequencing Data under Synchrotron Radiation. Transcriptomics. 2017;5:e117.

[81]. Heidari A. Elimination of the Heavy Metals Toxicity and Diseases in Disruption of Extracellular Matrix (ECM) Proteins and Cell Adhesion Intelligent Nanomolecules Adjustment in Cancer Metastases Using Metalloenzymes and under Synchrotron Radiation. Lett Health Biol Sci. 2017;2(2):1-4.

[82]. Heidari A. Treatment of Breast Cancer Brain Metastases through a Targeted Nanomolecule Drug Delivery System Based on Dopamine Functionalized Multi-Wall Carbon Nanotubes (MWCNTs) Coated with Nano Graphene Oxide (GO) and Protonated Polyaniline (PANI) in Situ During the Polymerization of Aniline Autogenic Nanoparticles for the Delivery of AntiCancer Nano Drugs under Synchrotron Radiation. Br J Res. 2017;4(3):16.

[83]. Heidari A. Sedative, Analgesic and Ultrasound-Mediated Gastrointestinal Nano Drugs Delivery for Gastrointestinal Endoscopic Procedure, Nano Drug-Induced Gastrointestinal Disorders and Nano Drug Treatment of Gastric Acidity. Res Rep Gastroenterol. 2017;1(1):1.

[84]. Heidari A. Synthesis, Pharmacokinetics, Pharmacodynamics, Dosing, Stability, Safety and Efficacy of Orphan Nano Drugs to Treat High Cholesterol and Related Conditions and to Prevent Cardiovascular Disease under Synchrotron Radiation. Journal of Pharmaceutical Sciences \& Emerging Drugs. 2017 Aug 22;2017.

[85]. Heidari A. Non-Linear Compact Proton Synchrotrons to Improve Human Cancer Cells and Tissues Treatments and Diagnostics through Particle Therapy Accelerators with Monochromatic Microbeams. J Cell Biol Mol Sci. 2017;2(1):1-5.

[86]. Heidari A. Design of Targeted Metal Chelation Therapeutics Nanocapsules as Colloidal Carriers and Blood-Brain Barrier (BBB) Translocation to Targeted Deliver Anti-Cancer Nano Drugs into the Human Brain to Treat Alzheimer's Disease under Synchrotron Radiation. J Nanotechnol Material Sci. 2017;4(2):1-5.

[87]. Gobato R, Heidari A. Calculations Using Quantum Chemistry for Inorganic Molecule Simulation BeLi2SeSi. American Journal of Quantum Chemistry and Molecular Spectroscopy. 2017;2(3):37-46.

[88]. Heidari A. Different High-Resolution Simulations of Medical, Medicinal, Clinical, Pharmaceutical and Therapeutics Oncology of Human Lung Cancer Translational Anti-Cancer Nano Drugs Delivery Treatment Process under Synchrotron and X-Ray Radiations. J Med Oncol. 2017;1(1):1.

[89]. Heidari A. A Modern Ethnomedicinal Technique for Transformation. Prevention and Treatment of Human Malignant Gliomas Tumors into Human Benign Gliomas Tumors under Synchrotron Radiation. Am J Ethnomed. 2017;4(1):10

[90]. Heidari A. An investigation of the role of DNA as molecular computers: A computational study on the Hamiltonian path problem. International Journal of Scientific \& Engineering Research. 2014;5(1):1884-9.

[91]. Heidari A. Active Targeted Nanoparticles for Anti-Cancer Nano Drugs Delivery across the Blood-Brain Barrier for Human Brain Cancer Treatment. Multiple Sclerosis (MS) and Alzheimer's Diseases Using Chemical Modifications of Anti-Cancer Nano Drugs or Drug-Nanoparticles through Zika Virus (ZIKV) Nanocarriers under Synchrotron Radiation. J Med Chem Toxicol. 2017;2(3):1-5.

[92]. Heidari A. Investigation of Medical, Medicinal, Clinical and Pharmaceutical Applications of Estradiol, Mestranol (Norlutin), Norethindrone (NET), Norethisterone Acetate (NETA), Norethisterone Enanthate (NETE) and Testosterone Nanoparticles as Biological Imaging, Cell Labeling, AntiMicrobial Agents and Anti-Cancer Nano Drugs in Nanomedicines Based Drug Delivery Systems for Anti-Cancer Targeting and Treatment. Parana Journal of Science and Education (PJSE). 2017;3(4):10-9.

[93]. Heidari A. A Comparative Computational and Experimental Study on Different Vibrational Biospectroscopy Methods, Techniques and Applications for Human Cancer Cells in Tumor Tissues Simulation, Modeling, Research, Diagnosis and Treatment. Open J Anal Bioanal Chem. 2017;1(1):014-20.

[94]. Heidari A. Combination of DNA/RNA Ligands and Linear/Non-Linear Visible-Synchrotron Radiation-Driven N-Doped Ordered Mesoporous Cadmium Oxide (CdO) Nanoparticles Photocatalysts Channels Resulted in an Interesting Synergistic Effect Enhancing Catalytic Anti-Cancer Activity. Enz Eng. 2017;6(1).

[95]. Heidari A. Modern Approaches in Designing Ferritin, Ferritin Light Chain, Transferrin, Beta-2 Transferrin and Bacterioferritin-Based Anti-Cancer Nano Drugs Encapsulating Nanosphere as DNA-Binding Proteins from Starved Cells (DPS). Mod Appro Drug Des. 2017;1(1):000504.

[96]. Heidari A. Potency of Human Interferon $\beta-1 \mathrm{a}$ and Human Interferon $\beta-1 \mathrm{~b}$ 
in Enzymotherapy, Immunotherapy, Chemotherapy, Radiotherapy, Hormone Therapy and Targeted Therapy of Encephalomyelitis Disseminate/ Multiple Sclerosis (MS) and Hepatitis A, B, C, D, E, F and G Virus. J Proteomics Enzymol. 2017;6(1).

[97]. Heidari A. Transport Therapeutic Active Targeting of Human Brain Tumors Enable Anti-Cancer Nanodrugs Delivery across the Blood-Brain Barrier (BBB) to Treat Brain Diseases Using Nanoparticles and Nanocarriers under Synchrotron Radiation. J Pharm Pharmaceutics. 2017;4(2):1-5.

[98]. Heidari A, Brown C. Combinatorial Therapeutic Approaches to DNA/RNA and Benzylpenicillin (Penicillin G), Fluoxetine Hydrochloride (Prozac and Sarafem), Propofol (Diprivan), Acetylsalicylic Acid (ASA)(Aspirin), Naproxen Sodium (Aleve and Naprosyn) and Dextromethamphetamine Nanocapsules with Surface Conjugated DNA/RNA to Targeted Nano Drugs for Enhanced Anti-Cancer Efficacy and Targeted Cancer Therapy Using Nano Drugs Delivery Systems. Ann Adv Chem. 2017;1(2):061-9.

[99]. Heidari A. Vibrational Spectroscopy of Nucleic Acids", Wahid Ali Khan (Editor), "Basic Biochemistry”. 2016: 1-18.

[100]. Heidari A. High-resolution simulations of human brain cancer translational nano drugs delivery treatment process under synchrotron radiation. J Transl Res. 2018;1(1).

[101]. Heidari A. Investigation of Anti-Cancer Nano Drugs' Effects' Trend on Human Pancreas Cancer Cells and Tissues Prevention, Diagnosis and Treatment Process under Synchrotron and X-Ray Radiations with the Passage of Time Using Mathematica. Current Trends Anal Bioanal Chem 1 (1):36-41.

[102]. Heidari A. Pros and Cons Controversy on Molecular Imaging and Dynamics of Double-Standard DNA/RNA of Human Preserving Stem Cells-Binding Nano Molecules with Androgens/Anabolic Steroids (AAS) or Testosterone Derivatives through Tracking of Helium-4 Nucleus (Alpha Particle) Using Synchrotron Radiation. Arch Biotechnol Biomed. 2017;1(1):067-100.

[103]. Heidari A. Visualizing Metabolic Changes in Probing Human Cancer Cells and Tissues Metabolism Using Vivo 1H or Proton NMR, 13C NMR, 15N NMR and 31P NMR Spectroscopy and Self-Organizing Maps under Synchrotron Radiation. SOJ Mater Sci Eng. 2017;5(2):1-6.

[104]. Heidari A. Cavity Ring-Down Spectroscopy (CRDS), Circular Dichroism Spectroscopy, Cold Vapour Atomic Fluorescence Spectroscopy and Correlation Spectroscopy Comparative Study on Malignant and Benign Human Cancer Cells and Tissues with the Passage of Time under Synchrotron Radiation. Enliven: Challenges Cancer Detect Ther. 2017;4(2):e001.

[105]. Heidari A. Laser Spectroscopy, Laser-Induced Breakdown Spectroscopy an d LaserInduced Plasma Spectroscopy Comparative Study on Malignant and Benign Human Cancer Cells and Tissues with the Passage of Time under Synchrotron Radiation. Int J Hepatol Gastroenterol. 2017;3(4):079-84.

[106].Heidari A. Time-Resolved Spectroscopy and Time-Stretch Spectroscopy Comparative Study on Malignant and Benign Human Cancer Cells and Tissues with the Passage of Time under Synchrotron Radiation. Enliven: Pharmacovigilance and Drug Safety. 2017;4(2):e001.

[107]. Heidari A. Overview of the Role of Vitamins in Reducing Negative Effect of Decapeptyl (Triptorelin Acetate or Pamoate Salts) on Prostate Cancer Cells and Tissues in Prostate Cancer Treatment Process through Transformation of Malignant Prostate Tumors into Benign Prostate Tumors under Synchrotron Radiation. Open J Anal Bioanal Chem. 2017;1(1):021-6.

[108]. Alireza Heidari, "Electron Phenomenological Spectroscopy, Electron Paramagnetic Resonance (EPR) Spectroscopy and Electron Spin Resonance (ESR) Spectroscopy Comparative Study on Malignant and Benign Human Cancer Cells and Tissues with the Passage of Time under Synchrotron Radiation", Austin J Anal Pharm Chem. 2017; 4(3):1091.

[109]. Heidari A. Therapeutic Nanomedicine Different High-Resolution Experimental Images and Computational Simulations for Human Brain Cancer Cells and Tissues Using Nanocarriers Deliver DNA/RNA to Brain Tumors under Synchrotron Radiation with the Passage of Time Using Mathematica and MATLAB. Madridge J Nano Tech. 2017 Nov 24;2(2):77-83.

[110]. Heidari A. A Consensus and Prospective Study on Restoring Cadmiu m Oxide (CdO) Nanoparticles Sensitivity in Recurrent Ovarian Cancer b y Extending the Cadmium Oxide (CdO) Nanoparticles-Free Interval Usin g Synchrotron Radiation Therapy as Antibody-Drug Conjugate for the Treatment of Limited-Stage Small Cell Diverse Epithelial Cancers. Cancer Clin Res Rep. 2017;2.

[111]. Heidari A. A Novel and Modern Experimental Imaging and Spectroscopy Comparative Study on Malignant and Benign Human Cancer Cells and Tissues with the Passage of Time under White Synchrotron Radiation. Cancer Sci Res Open Access. 2017;4(2):1-8.

[112]. Heidari A. Different High-Resolution Simulations of Medical, Medicinal, Clinical, Pharmaceutical and Therapeutics Oncology of Human Lung Cancer Translational Anti-Cancer Nano Drugs Delivery Treatment Process under Synchrotron and X-Ray Radiations. J Med Oncol. 2017;1(1):1.

[113]. Heidari A. Vibrational Decihertz $(\mathrm{dHz})$, Centihertz $(\mathrm{cHz})$, Millihertz $(\mathrm{mHz})$, Microhertz $(\mu \mathrm{Hz})$, Nanohertz $(\mathrm{nHz})$, Picohertz $(\mathrm{pHz})$, Femtohertz
$(\mathrm{fHz})$, Attohertz $(\mathrm{aHz})$, Zeptohertz $(\mathrm{zHz})$ and Yoctohertz $(\mathrm{yHz})$ Imaging and Spectroscopy Comparative Study on Malignant and Benign Human Cancer Cells and Tissues under Synchrotron Radiation. J Biomed. 2017 Dec 1;7(4):335-40.

[114].Heidari A. Force Spectroscopy and Fluorescence Spectroscopy Comparative Study on Malignant and Benign Human Cancer Cells and Tissues with the Passage of Time under Synchrotron Radiation. EC Cancer. 2017;2:239-46.

[115]. Heidari A. Photoacoustic Spectroscopy, Photoemission Spectroscopy and Photothermal Spectroscopy Comparative Study on Malignant and Benign Human Cancer Cells and Tissues with the Passage of Time Under Synchrotron Radiation. BAOJ Cancer Res Ther. 2017 3: 045.

[116].Heidari A. J-Spectroscopy, Exchange Spectroscopy (EXSY), Nucle ᄀ ar Overhauser Effect Spectroscopy (NOESY) and Total Correlation Spectroscopy (TOCSY) Comparative Study on Malignant and Benign HumanCancer Cells and Tissues under Synchrotron Radiation. EMS Eng Sci J. 2017;1(2):006-13.

[117]. Heidari A. Neutron Spin Echo Spectroscopy and Spin Noise Spectroscopy Comparative Study on Malignant and Benign Human Cancer Cells and Tissues with the Passage of Time under Synchrotron Radiation. Int J Biopharm Sci. 2018;1:103

[118]. Heidari A. Vibrational Decahertz (daHz), Hectohertz (hHz), Kilohertz $(\mathrm{kHz})$, Megahertz $(\mathrm{MHz})$, Gigahertz $(\mathrm{GHz})$, Terahertz $(\mathrm{THz})$, Petahertz $(\mathrm{PHz})$, Exahertz $(\mathrm{EHz})$, Zettahertz $(\mathrm{ZHz})$ and Yottahertz $(\mathrm{YHz})$ Imaging and Spectroscopy Comparative Study on Malignant and Benign Human Cancer Cells and Tissues under Synchrotron Radiation. Madridge J Anal Sci Instrum. 2017;2(1):41-46.

[119].Heidari A. Two-Dimensional Infrared Correlation Spectroscopy, Linear TwoDimensional Infrared Spectroscopy and Non-Linear Two-Dimensional Infrared Spectroscopy Comparative Study on Malignant and Benign $\mathrm{Hu}-$ man Cancer Cells and Tissues under Synchrotron Radiation with the Passage of Time. J Mater Sci Nanotechnol. 2018;6(1): 101.

[120]. Heidari A. Fourier Transform Infrared (FTIR) Spectroscopy, Near-Infrared Spectroscopy (NIRS) and Mid-Infrared Spectroscopy (MIRS) Comparative Study on Malignant and Benign Human Cancer Cells and Tissues under Synchrotron Radiation with the Passage of Time. Int J Nanotechnol Nanomed. 2018;3(1):1-6.

[121].Alireza Heidari, "Infrared Photo Dissociation Spectroscopy and Infrared Correlation Table Spectroscopy Comparative Study on Malignant and Benign Human Cancer Cells and Tissues under Synchrotron Radiation with the Passage of Time", Austin Pharmacol Pharm. 2018;3(1): 1011

[122]. Heidari A. Novel and Transcendental Prevention, Diagnosis and Treatment Strategies for Investigation of Interaction among Human Blood Cancer Cells, Tissues, Tumors and Metastases with Synchrotron Radiation under Anti-Cancer Nano Drugs Delivery Efficacy Using MATLAB Modeling and Simulation. Madridge J Nov Drug Res. 2017;1(1):18-24.

[123]. Heidari A. Comparative Study on Malignant and Benign Human Cancer Cells and Tissues with the Passage of Time under Synchrotron Radiation. Open Access J Trans Med Res. 2018;2(1):00026-00032.

[124]. Marcia G, Ricardo G, Heidari A. Planting of Jaboticaba Trees for Landscape Repair of Degraded Area. Landscape Architecture and Regional Planning. 2018;3(1):1-9.

[125]. Heidari A. Correlation Two-Dimensional Nuclear Magnetic Resonance (NMR) (2D-NMR) (COSY) Imaging and Spectroscopy Comparative Study on Malignant and Benign Human Cancer Cells and Tissues under Synchrotron Radiation. EMS Cancet Sci J. 2018;1(1):001.

[126]. Heidari A. Fluorescence Spectroscopy, Phosphorescence Spectroscopy and Luminescence Spectroscopy Comparative Study on Malignant and Benign Human Cancer Cells and Tissues under Synchrotron Radiation with the Passage of Time. SM J Clin. Med. 2018;4(1):1018.

[127].Heidari A. Nuclear Inelastic Scattering Spectroscopy (NISS) and Nuclear Inelastic Absorption Spectroscopy (NIAS) Comparative Study on Malignant and Benign Human Cancer Cells and Tissues under Synchrotron Radiation. Int J Pharm Sci. 2018;2(1):1-14.

[128]. Heidari A. X-Ray Diffraction (XRD), Powder X-Ray Diffraction (PXRD) and Energy-Dispersive X-Ray Diffraction (EDXRD) Comparative Study on Malignant and Benign Human Cancer Cells and Tissues under Synchrotron Radiation. J Oncol Res. 2018;2(1):1-14.

[129]. Heidari A. Thermal Spectroscopy, Photothermal Spectroscopy, Thermal Microspectroscopy, Photothermal Microspectroscopy, Thermal Macrospectroscopy and Photothermal Macrospectroscopy Comparative Study on Malignant and Benign Human Cancer Cells and Tissues with the Passage of Time under Synchrotron Radiation. SM J Biometrics Biostat. 2018; 3(1):1024

[130].Heidari A. A Modern and Comprehensive Experimental Biospectroscopic Comparative Study on Human Common Cancers' Cells, Tissues and Tumors before and after Synchrotron Radiation Therapy. Open Acc J Oncol Med. 2018;1(1).

[131].Heidari A. Heteronuclear Correlation Experiments such as Heteronuclear 
Single-Quantum Correlation Spectroscopy (HSQC), Heteronuclear Multiple-Quantum Correlation Spectroscopy (HMQC) and Heteronuclear Multiple-Bond Correlation Spectroscopy (HMBC) Comparative Study on Malignant and Benign Human Endocrinology and Thyroid Cancer Cells and Tissues under Synchrotron Radiation. J Endocrinol Thyroid Res. 2018;3(1):555603.

[132]. Heidari A. Nuclear Resonance Vibrational Spectroscopy (NRVS), Nuclear Inelastic Scattering Spectroscopy (NISS), Nuclear Inelastic Absorption Spectroscopy (NIAS) and Nuclear Resonant Inelastic X-Ray Scattering Spectroscopy (NRIXSS) Comparative Study on Malignant and Benign Human Cancer Cells and Tissues under Synchrotron Radiation. Int J Bioorg Chem Mol Biol. 2018 Feb 7;6(1e):1-5.

[133]. Heidari A. A Novel and Modern Experimental Approach to Vibrational Circular Dichroism Spectroscopy and Video Spectroscopy Comparative Study on Malignant and Benign Human Cancer Cells and Tissues with the
Passage of Time under White and Monochromatic Synchrotron Radiation. Glob J Endocrinol Metab. 2018;1(3):000514-000519.

[134].Heidari A. Pros and Cons Controversy on Heteronuclear Correlation Experiments such as Heteronuclear Single-Quantum Correlation Spectroscopy (HSQC), Heteronuclear Multiple-Quantum Correlation Spectroscopy (HMQC) and Heteronuclear Multiple-Bond Correlation Spectroscopy (HMBC) Comparative Study on Malignant and Benign Human Cancer Cells and Tissues under Synchrotron Radiation. EMS Pharma J. 2018;1(1):002-008.

[135]. Heidari A. A Modern Comparative and Comprehensive Experimental Biospectroscopic Study on Different Types of Infrared Spectroscopy of Malignant and Benign Human Cancer Cells and Tissues with the Passage of Time under Synchrotron Radiation. J Analyt Molecul Tech. 2018;3(1):8. 